\title{
CD25 Negative
}

National Cancer Institute

\section{Source}

National Cancer Institute. CD25 Negative. NCI Thesaurus. Code C133815.

An indication that CD25 expression was not detected in a sample. 